\title{
Unlocking the Climate Record Stored within Mars' Polar Layered Deposits
}

\section{Authors and Affiliations}

Isaac B. Smith

York University, Toronto, Ontario, Canada, M3J 1P3

Planetary Science Institute, Lakewood, CO 80401

Paul O. Hayne

University of Colorado Boulder, Boulder, Colorado 80309

Shane Byrne

University of Arizona, Tucson, Arizona 85721

Patricio Becerra

Universität Bern, Bern, Switzerland

Melinda Kahre

NASA Ames Research Center, Moffett Field, California 94035

Wendy Calvin

University of Nevada, Reno, Reno, Nevada 89557

Christine Hvidberg

University of Copenhagen, Copenhagen, Denmark

Sarah Milkovich

Jet Propulsion Laboratory, California Institute of Technology, Pasadena, California 91109

Peter Buhler

Jet Propulsion Laboratory, California Institute of Technology, Pasadena, California 91109

Margaret Landis

University of Colorado Boulder, Boulder, Colorado 80309

Briony Horgan

Purdue University, West Lafayette, Indiana 47907

Armin Kleinböhl

Jet Propulsion Laboratory, California Institute of Technology, Pasadena, California 91109

Matthew R. Perry

Planetary Science Institute, Lakewood, CO 80401

Rachel Obbard

SETI Institute, Mountain View, California 94043

Jennifer Stern

NASA Goddard Space Flight Center, Greenbelt, Maryland 20771

Sylvain Piqueux

Jet Propulsion Laboratory, California Institute of Technology, Pasadena, California 91109

Nicolas Thomas

Universität Bern, Bern, Switzerland

Kris Zacny

Honeybee Robotics, Pasadena, California 91103

Lynn Carter

University of Arizona, Tucson, Arizona 85721

Lauren Edgar

U.S. Geological Survey, Flagstaff, Arizona 86001 
Jeremy Emmett

New Mexico State University, Las Cruces, New Mexico 88003

Thomas Navarro

McGill Space Institute, McGill University, Montréal, Québec, Canada

University of California, Los Angeles, Los Angeles, California 90095

Jennifer Hanley

Lowell Observatory, Flagstaff, Arizona 86001

Michelle Koutnik

University of Washington, Seattle, Washington 98195

Nathaniel Putzig

Planetary Science Institute, Lakewood, CO 80401

Bryana L. Henderson

Jet Propulsion Laboratory, California Institute of Technology, Pasadena, California 91109 John W. Holt

University of Arizona, Tucson, Arizona 85721

Bethany Ehlmann

California Institute of Technology, Pasadena, California 91125

Jet Propulsion Laboratory, California Institute of Technology, Pasadena, California 91109

Sergio Parra

California Institute of Technology, Pasadena, California 91125

Daniel Lalich

Cornell University, Ithaca, New York 14853

Candice Hansen

Planetary Science Institute, Tucson, Arizona 85719

Michael Hecht

Haystack Observatory, Westford, Massachusetts 01886

Don Banfield

Cornell University, Ithaca, New York 14853

Ken Herkenhoff

U.S. Geological Survey, Flagstaff, Arizona 86001

David A. Paige

University of California, Los Angeles, Los Angeles, California 90095

Mark Skidmore

Montana State University, Bozeman, Montana 59717

Robert L. Staehle

Jet Propulsion Laboratory, California Institute of Technology, Pasadena, California 91109

Matthew Siegler

Planetary Science Institute, Tucson, Arizona 85719 


\section{Signatories}
A. Soto
Southwest Research Institute
asoto@boulder.swri.edu
F. Foss
Freestyle Analytical \& Quantitative Service LLC, foss@airmail.net
S. Lewis
The Open University UK
J. Whitten
Tulane University
E. Vos
Weizmann Institute of Science
stephen.lewis@open.ac.uk
P. A. Johnson
University of Alberta
J. C. Johnson
University of Alberta
C. Gallagher
University College Dublin
A. Brown
Plancius Research
T. Bertrand
NASA Ames Research Center
jwhitten1@tulane.edu
C. Phillips-Lander Southwest Research Institute
Eran.Vos@weizmann.ac.il
N. Oliveira
Valongo Observatory
E. Kite
University of Chicago
T. Thorsteinsson
L. Tamppari
Icelandic Meteorological Office
paj1@ualberta.ca
jcj2@ualberta.ca
colman.gallagher@ucd.ie
Adrian.J.Brown@nasa.go
tanguy.bertrand@nasa.gov
charity.lander@gmail.com
natanael18@astro.ufrj.br
kite@uchicago.edu
E. Hauber
Jet Propulsion Laboratory
L. Fanara
German Aerospace Center (DLR)
thor@vedur.is
leslie.k.tamppari@jpl.nasa.gov
ernst.hauber@dlr.de
J. Oberst
German Aerospace Center (DLR)
Lida.Fanara@dlr.de
S. Ulamec
German Aerospace Center (DLR)
S. Cartwright
German Aerospace Center (DLR)
juergen.oberst@dlr.de
T. Harrison
University of Reno Nevada
S. Hibbard
Planet Federal
A. Portyankina
University of Western Ontario
Stephan.Ulamec@dlr.de
scartwright@nevada.unr.edu
tanya.harrison@federal.planet.com
shibbard@uwo.ca
T. N. Titus
University of Colorado LASP
Ganna.Portyankina@lasp.colorado.edu
U.S. Geological Survey
ttitus@usgs.gov 


\section{Executive Summary}

In the icy beds of its polar layered deposits (PLD), Mars likely possesses a record of its recent climate history, analogous to terrestrial ice sheets that contain records of Earth's past climate. Both northern and southern PLDs store information on the climatic and atmospheric state during the deposition of each layer (WPs: Becerra et al.; Smith et al). Reading the climate record stored in these layers requires detailed measurements of layer composition, thickness, isotope variability, and near-surface atmospheric measurements. We identify four fundamental questions that must be answered in order to interpret this climate record and decipher the recent climatic history of Mars:

1. Fluxes: What are the present and past fluxes of volatiles, dust, and other materials into and out of the polar regions?

2. Forcings: How do orbital/axial forcing and exchange with other reservoirs affect those fluxes?

3. Layer Processes: What chemical and physical processes form and modify layers?

4. Record: What is the timespan, completeness, and temporal resolution of the climate history recorded in the PLD?

In a peer reviewed report (1), we detailed a sequence of missions, instruments, and architecture needed to answer these questions. Here, we present the science drivers and a mission concept for a polar lander that would enable a future reading of the past few million years of the Martian climate record. The mission addresses as-yet-unachieved science goals of the current Decadal Survey and of MEPAG for obtaining a record of Mars climate and has parallel goals to the NEXSAG and ICE-SAG reports.

\section{Overview of the Martian Polar Layered Deposits}

The layered structure of the PLD can be seen through visible imaging of bed exposures in troughs and scarps (Figures 1,2) and radar sounding data (Figure 3). Variations in the orbital and axial configuration of Mars likely led to climatic changes that formed the layers we see (2) including multiple angular unconformities in the stratigraphic record that show periods of net ablation and accumulation (3). Extremely dusty layers may be sublimation lag deposits that represent disconformities in the record (4). In addition, the PLDs are partly covered with bright residual ice caps that strongly affect the current climate (Figure 1). The north polar residual cap interacts with the atmosphere and records present day processes - to become a future layer in the climate archive.

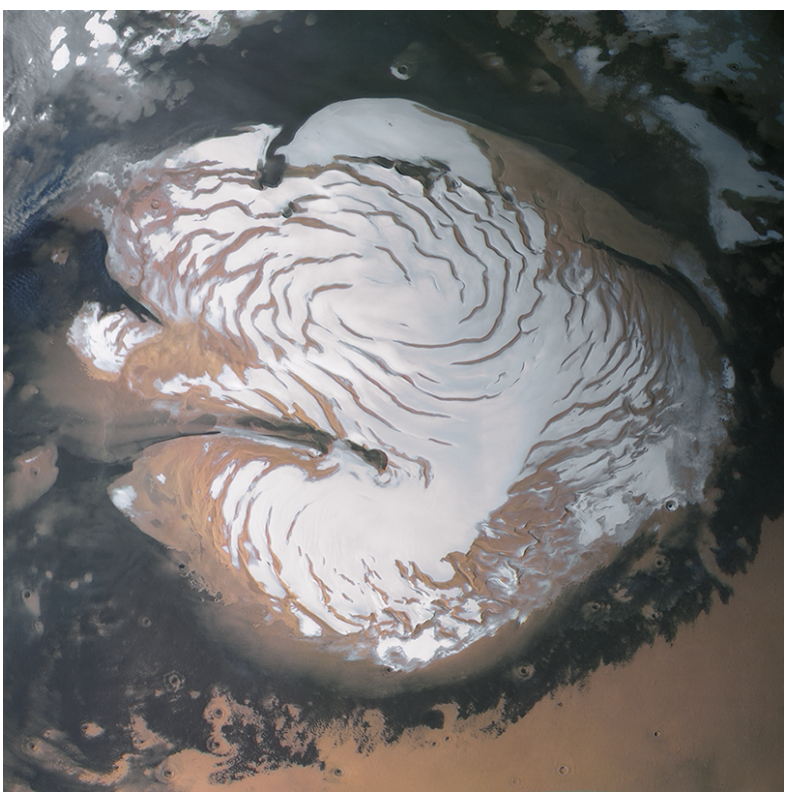

The gross and net annual exchange of $\mathrm{H}_{2} \mathrm{O}$ onto Figure 1: The NPLD imaged by ESA's High either polar cap is unknown, but the net flux is Resolution Stereo Camera in late spring thought to average $0.5 \mathrm{~kg} \mathrm{~m}^{-2} \mathrm{y}^{-1}$ over the last few without seasonal frost cover. The NPLD has Myr onto the North PLD (NPLD). This unknown a surficial dust lag, but much of the bright mass balance value is a key parameter to understand north polar residual cap is uncovered. The the current climate, so knowledge of this value will residual cap provides the majority of the help tune and validate global climate models atmospheric water vapor of Mars each year. 


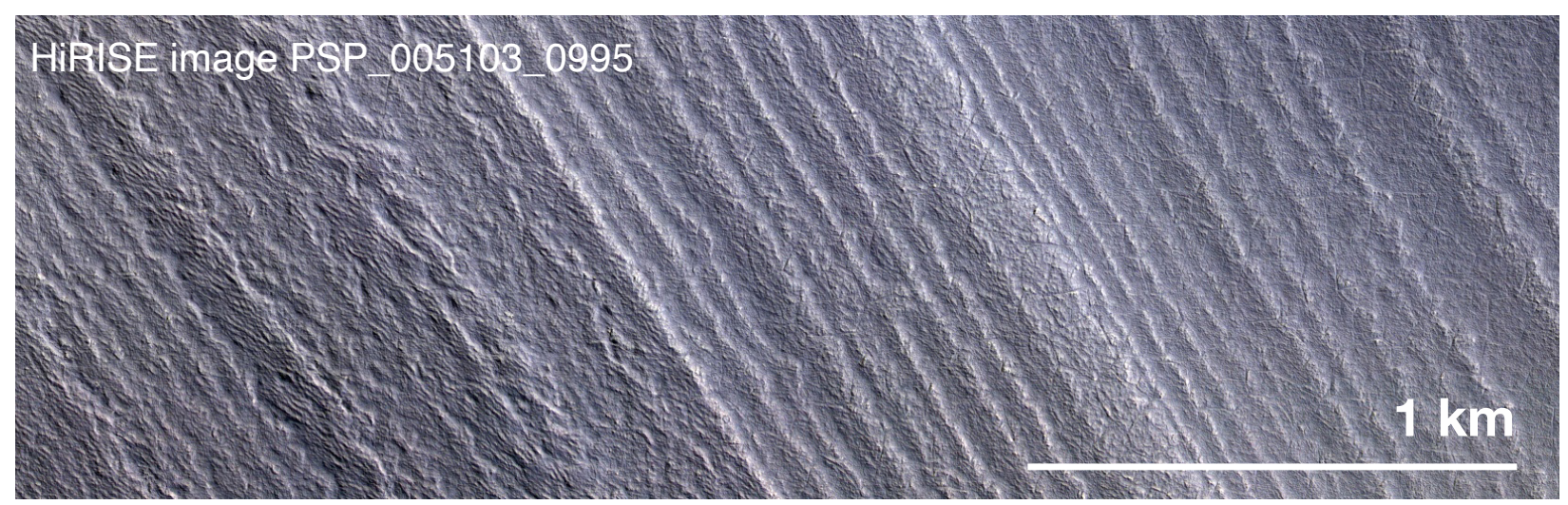

Figure 2: Layers in the South PLD. Exposed layers imply recent erosion; however, a dust lag may slow sublimation on annual time scales.

(GCMs) of modern climate, supporting their application to understanding past climatic states. In this white paper, we focus on the NPLD, for which hypotheses are much more robust.

Layer Composition: During orbital/axial variations, the transfer of ice, aerosols, and dust from lower latitudes to the poles forms beds with variable ice content. The bulk composition of the NPLD is $\sim 95 \%$ water ice (5). Besides ice, PLD composition likely includes dust, salts, other lithic materials, gas bubbles, HDO (deuterated water), and daughter products of radioactive decay, in decreasing order by volume. As on Earth, these components can be used to infer sintering rate, dust/ice ratio, and concentrations of different isotopes in ice, dust, and trapped gases.

Individual beds may be up to $50 \%$ dust (6). These dust-rich beds may have formed during periods of high dust accumulation or during periods of ice loss and lag formation (7). Dust-poor beds must have formed during times when ice deposition was high relative to dust accumulation. The stratified variability of each material represents the climate archive.

In order to detect climate signals, we need to be able to observe and describe intrinsic properties of the beds that make up the record, i.e., properties that relate most closely to the environmental conditions at the time of accumulation.

Forcings: Three astronomical cycles drive most of Mars' climate variations: obliquity, argument of perihelion, and eccentricity. These cycles affect the average solar flux at the Martian surface and drive the stability of ice at various locations on the planet. Other external forcings of Mars' climate include solar wind, Jeans escape, cosmic rays, impactors, etc., that act to strip volatiles away from the upper atmosphere or, on smaller scales, implant new volatiles. Secular mass loss driven by the solar wind put Mars into its current, low-pressure state billions of years ago (8). On timescales relevant to the polar ice, atmospheric stripping may have affected fractionation rates for isotopes in parallel with solar cycles (9). The major intrinsic climate driver is the poorly understood dust cycle, an important topic for future investigation (WP: Newman et al.).

Isotope Variability in Layers: PLD layers likely retain a combination of ices $\left(\mathrm{H}_{2} \mathrm{O}, \mathrm{CO}_{2}\right)$, dust, and trapped gasses. Each may vary in isotopic composition due to fractionation processes. The ratio of deuterium to hydrogen $(\mathrm{D} / \mathrm{H})$ in PLD water ice is an invaluable data point. This composition can tell us about variations in obliquity, which in turn provides information on what reservoirs are accessed at different axial states (e.g., $(9,10))$.

Layer Formation: Investigating layer formation is critical to reading the stratigraphic climate record that is made up of these layers. Thus, we need to measure the processes forming the current 


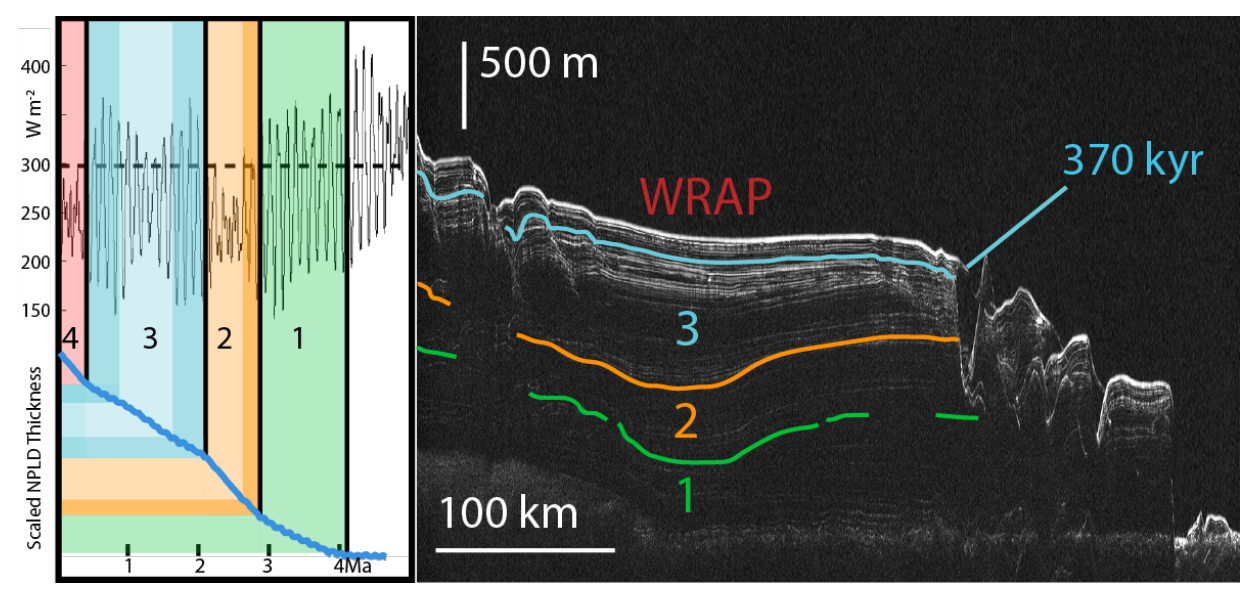

Figure 3: Comparison of model from (11) (left) to radar stratigraphic mapping of the NPLD (right). Model calculates solar insolation in $\mathrm{Wm}^{-2}$ starting at $\sim 4 \mathrm{Ma}$ and predicts four distinct periods of NPLD accumulation. Blue line in left panel is total accumulation of NPLD ice. Approximately 30 lag deposits are expected to derive from obliquity changes, similar in number to the total number of reflectors that SHARAD detects (right). The Widespread Recent Accumulation Package (WRAP) is associated with the current interglacial period (12).

layer that is expected to eventually be included in the climate archive. Many processes occur at the surface, including ice crystallization, dust deposition, isotope fractionation, and heat loss or gain. Processes continue even after burial, such as sintering, phase partitioning, and impurity exclusion. Observing these processes is today's well-characterized climate would allow us to connect deeper layer properties to past climates.

\section{Major Questions and Observables}

Fluxes: Measurements of the modern-day fluxes of volatiles, dust, and other materials from lower latitudes to the polar regions and then onto the polar caps are key steps to interpreting the climate record in the PLDs. These would link observable modern climatic processes to material deposition at the PLD surface, and thereby layer formation. We must measure fluxes at global and regional scales and the exchange between the surface and atmosphere. Several observations are necessary from the surface: 1) wind speed profile near the surface boundary layer to provide input for calculating regional flux; 2) horizontal and vertical wind speeds within the planetary boundary layer; 3) dust and condensate concentrations in the planetary boundary layer; 4) vertical in situ surface mass flux of $\mathrm{H}_{2} \mathrm{O}$, refractory material, and $\mathrm{CO}_{2}$.

Meteorologic measurements at the surface should be compared to global and regional measurements in order to determine the availability of transportable material through the present epoch. Similar to layer formation, measuring meteorological processes requires in situ observation over an entire martian year (ICE-SAG). Measurements should include the ablation and/or accumulation of dust and ice $\left(\mathrm{H}_{2} \mathrm{O}\right.$ and $\left.\mathrm{CO}_{2}\right)$, saltation and surface transport of materials, wind speed, insolation, albedo, and temperature, pressure, and humidity (TPH).

Layer-Formation Processes: Determining all of the processes that affect layers is a critical knowledge gap. To characterize modern bed-formation processes, we need access to the surface and the upper $50 \mathrm{~cm}$ of the subsurface. This is expected to represent the last 1000 years of Mars history (which are expected to have experienced similar climatic and surface processes to the present day) and should capture all of the processes actively affecting layer formation. Impurity 
composition, abundance, and distribution are the top priority measurements. Additional items to measure are phase partitioning of salt and other impurities from ice grains; density and grain size distribution of dust, along with isotope fraction.

Age Dating the NPLD: Measuring ages hinges on finding datable lithic material in the form of dust, volcanic ash, or impact ejecta that are accessible to robotic explorers. This can be done by measuring decay products of radioactive elements (e.g. He, Ne, Be, Ar) and the concentrations of cosmogenic radionuclides. Extremely high precision measurements are required in order to achieve useful ages because of the youthfulness and low lithic content of the NPLD, with age resolution on the order of $10^{4}-10^{5}$ years. A highly selective and sensitive mass spectrometer similar in size, mass, and power to the SAM instrument on MSL can compete the task. Additionally, resonance ionization mass spectrometry (RIMS) is a promising technology for this purpose.

\section{Key Properties and Measurement Types:}

Flux measurements at the surface will quantify deposition and ablation processes. High frequency sampling of wind speed and direction in combination with TPH is necessary. Heat and momentum fluxes can be derived from these measurements. Airborne dust and ice particles should be counted in aggregate to distinguish particle type (e.g., dust, $\mathrm{CO}_{2}$ ice, and water ice). With hourly wind measurements, particulate measurements would enable the determination of dust and water ice particle transport through turbulent eddies. Depositional mass and dust-to-ices ratios are also required with microgram sensitivity. Measurement requirements and potential technologies are summarized in Table 1.

Layer formation: At the surface, determining the ratio of present-day accumulation of dust to $\mathrm{H}_{2} \mathrm{O}$ to $\mathrm{CO}_{2}$ is exceptionally important. A thermogravimeter with microgram accuracy can accumulate materials from snowfall and direct condensation, and then measure their mass before heating the volatiles to the appropriate temperature for sublimation. These measurements will have to be made throughout a full year, especially during polar winter. This is a key measurement to ground truth orbital measurements of grain size using emissivity (ICE-SAG).

Measurements of surface albedo, texture, grain size (up to 100 microns), grain density, and grain orientation should be made by reflectance infrared spectroscopy, a high-resolution imager, and nephelometry. Surface temperature, for calculating the thermal balance at the surfaceatmosphere interface, can be measured with a thermal imager.

Understanding layer formation also requires access to the near subsurface via drilling. Once a borehole is drilled, an optical imager could be lowered into the borehole in order to identify and count distinguishable layers and measure layer thickness. We expect that a resolution finer than 1 $\mathrm{mm}$ will be required to discern annual layers within the ice. A pixel scale of $\sim 30 \mu \mathrm{m}$ would suffice.

To characterize impurity distribution, miniaturized computerized tomography (microCT) is an excellent option. MicroCT collects axial X-ray scans and achieves a spatial resolution down to several microns, determining particle size and shape; volume concentration; pore size, shape, and distribution, discrimination of salts from Fe-rich sediment; and potentially relative atomic weight. MicroCT has been used recently in terrestrial ice analyses $(13,14)$. Measurements should require 
Table 1: Measurement requirements and potential technologies for in-situ measurements. Possible locations of each instrument on the spacecraft are: Lander Deck Atmospheric Instruments (LDA), Lander Deck Surface Measurements (LDS), Borehole Measurements (BH), Lander Deck Cuttings Measurements (LDC)

\begin{tabular}{|c|c|c|c|c|}
\hline Measurement & Sensitivity & Frequency & Location & Potential technology \\
\hline $\begin{array}{l}\text { Wind (near } \\
\text { surface) }\end{array}$ & $10 \mathrm{~cm} / \mathrm{s}$ & $20 \mathrm{~Hz}$ & LDA & Sonic anemometer \\
\hline Temperature & $0.5 \mathrm{~K}$ & $0.1 \mathrm{~Hz}$ & LDA & $\begin{array}{l}\text { Infrared Radiometer (e.g. } \\
\text { MEDA) }\end{array}$ \\
\hline Pressure & $1 \mathrm{~Pa}$ & $1 \mathrm{~Hz}$ & LDA & Barometer (e.g. MEDA) \\
\hline $\begin{array}{l}\text { Absolute } \\
\text { humidity }\end{array}$ & few ppm & $20 \mathrm{~Hz}$ & LDA & Atmospheric TLS (15) \\
\hline $\begin{array}{l}\text { Dust/water ice } \\
\text { flux }\end{array}$ & $\mu \mathrm{m}$ radius & $<1 \mathrm{~Hz}$ & LDA & Nephelometer \\
\hline Aerosols & $\mu \mathrm{m}$ radius & $<1 \mathrm{~Hz}$ & LDA & Nephelometer \\
\hline $\begin{array}{l}\text { Dust/water ice } \\
\text { accumulation }\end{array}$ & $<1 \mu \mathrm{g}$ & daily & LDS & $\begin{array}{c}\text { Micro-balance or } \\
\text { thermogravimeter } \\
\text { (evaporation capabilities) }\end{array}$ \\
\hline $\begin{array}{l}\text { Albedo, } \\
\text { texture, grains }\end{array}$ & $1 \mathrm{~mm}$ & Daily & LDS & $\begin{array}{l}\text { IR Spectroscopy, High } \\
\text { Resolution Imager, } \\
\text { Nephelometer }\end{array}$ \\
\hline Ice Strength & $1 \mathrm{~Pa}$ & $1 \mathrm{~mm}$ & $\mathrm{BH}$ & Drill Telemetry \\
\hline $\begin{array}{c}\text { Layer } \\
\text { Properties }\end{array}$ & $30 \mu \mathrm{m}$ & $1 \mathrm{~mm}$ & $\mathrm{BH}$ & Optical Imager in Borehole \\
\hline Organics & $\mathrm{ppm}$ to $\mathrm{ppb}$ & $1 \mathrm{~mm}$ & $\mathrm{BH}$ & XRF/APXS/LIBS/MOMA \\
\hline Salts & $0.1 \mathrm{wt} \%$ & $1 \mathrm{~mm}$ & $\mathrm{BH}$ & XRF/APXS/LIBS \\
\hline Trapped Gasses & $3,5,100 / \mathrm{mil}$ & $1 \mathrm{~mm}$ & LDC & MOMA \\
\hline $\begin{array}{l}\text { Impurity } \\
\text { Distribution }\end{array}$ & $\begin{array}{l}1 \mathrm{wt} \% \\
\sim 1 \mu \mathrm{m}\end{array}$ & $1 \mathrm{~mm}$ & LDC & micro CT \\
\hline
\end{tabular}

determining the mineralogy of refractory minerals such as dust and primary mafic sediments to a precision of $1 \mathrm{wt} \%$, distribution to $10 \mu \mathrm{m}$, and grain size for $1 \mu \mathrm{m}$ particles and larger.

Composition: Trapped gases, such as $\mathrm{CO}_{2}, \mathrm{~N}_{2}, \mathrm{Ar}, \mathrm{O}_{2}$, and $\mathrm{CO}$ are in sufficient abundance to measure at a precision of $0.1 \%$ or better, and minor constituents such as $\mathrm{NO}, \mathrm{Ne}, \mathrm{HDO}, \mathrm{Kr}$, and Xe require measurement precision of 10-100 ppb. Other important volatile species like $\mathrm{S}, \mathrm{Cl}$, and $\mathrm{CH}_{4}$ also require ppb precision. Bubble size and density should be measured to $10 \mu \mathrm{m}$. 
Isotopic measurements should include $\mathrm{D} / \mathrm{H}$ to a precision of $100 /$ mil over the range of 100 $9000 / \mathrm{mil},{ }^{18} \mathrm{O} /{ }^{16} \mathrm{O}$ at a precision of $3 / \mathrm{mil}$, and ${ }^{13} \mathrm{C} /{ }^{12} \mathrm{C}$ at a precision of $5 / \mathrm{mil}$. Concentrations of ${ }^{10} \mathrm{Be}$ are unknown, so determining if it is present in trace quantities will be valuable. Mass Spectroscopy is appropriate for those measurements. Composition and abundance of organics with a sensitivity of ppm to ppb is required. Similarly, determining the mineralogy and elemental composition of soluble impurities such as S/Cl-rich, perchlorate, and sulfate salts to a precision of $0.1 \mathrm{wt} \%$ is desired.

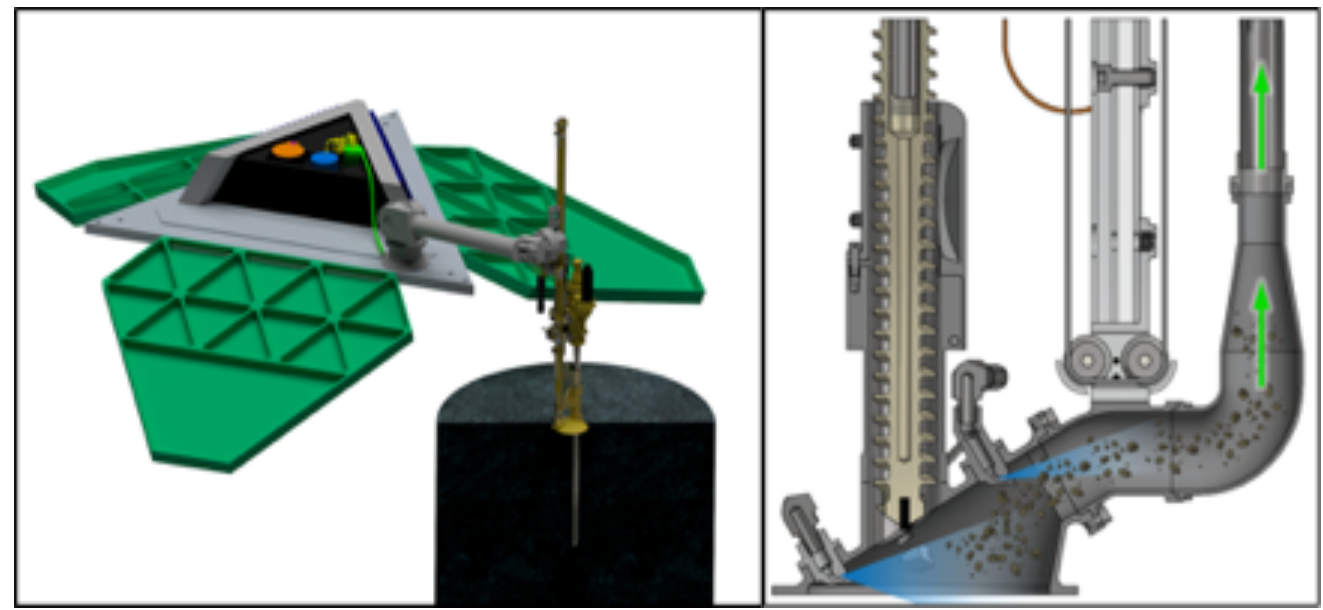

Figure 4: Concept of a lander asset using EDL architecture from MER. Left: A drill arm extends past the landing hardware and mechanically drills $\sim 50 \mathrm{~cm}$ into the subsurface using several small steps. Right: Pneumatic hardware moves the drill cuttings to the lander deck where they can be analyzed by onboard instruments.

\section{Mission Concept and Approaches to Measurements}

Static Lander: A mission to the NPLD surface should include instrumentation that is capable of addressing strategic knowledge gaps within the lower atmosphere, at the surface-atmosphere boundary, and within the top $50 \mathrm{~cm}$ of ice (Figure 4, Table 1). Many of the desired measurements require surviving or operating in the polar night, as discussed in the ICE-SAG report.

A meteorologic suite that accesses the bottom $4-5 \mathrm{~km}$ of the atmosphere would measure fluxes and boundary layer composition near the pole. TPH, and wind speed, including eddies, should be measured within $2 \mathrm{~m}$ of the surface. Imagers, a nephelometer, and a micro-balance would characterize the downward flux of material to the NPLD surface.

Borehole analysis. There are a few options for addressing the measurement requirements of the material within the borehole. The first option is to use the drill itself as an instrument to measure material strength and density from drilling telemetry, density from ultrasonic velocity, a temperature sensor, and bulk electrical resistivity (to detect "slush" formation). The second option is to modify the drill string to include instruments. Finally, a third technique is to lower instruments into the borehole after the drill string is removed.

Instruments that could be built into the drill string or lowered separately include an optical camera system and/or a LED-driven VNIR spectrometer system, a microscope, a spectrometer (such as Ma_MISS on ExoMars (16)), a fine-scale electrical resistivity probe, a temperature sensor, and a laser. The optical camera system should provide a wide field of view. The microscope should have a short working distance and be able to reach micron resolutions. An optical camera, 
spectrometer, and the microscope will require some illumination. In addition, we foresee the inclusion of a laser in the borehole as a source for performing Laser Induced Breakdown Spectroscopy (LIBS) and deep UV/Raman (17). The spectrometer would reside on the deck of the lander and be fed via fiber optic cable, while the laser would be integrated in the borehole logger. These instruments may measure $\mathrm{D} / \mathrm{H}$ or ${ }^{18} \mathrm{O} /{ }^{16} \mathrm{O}$.

Ice composition and ice grain size as well as the mineralogy of impurities could all be assessed rapidly via short-wave infrared reflectance spectroscopy (SWIR; 1.0-4.0 microns), which has been shown via orbital remote sensing to be highly sensitive to key phases in the NPLD and has been successfully miniaturized for small applications.

Bulk chemistry of borehole walls or other solid samples could be assessed via X-ray fluorescence (XRF), as implemented in the Planetary Instrument for X-ray Lithochemistry (PIXL) instrument on Mars 2020; via alpha particle X-ray spectroscopy (APXS), as implemented on multiple Mars rovers; or via laser-induced breakdown spectroscopy (LIBS) - as implemented in the MSL ChemCam and Mars 2020 SuperCam instruments. LIBS has been implemented with long fiber optic cables and may be better suited to small diameter boreholes.

Sample analysis on the lander deck: This may be done using a single core, drill cuttings, melting the sample and transporting it as a liquid, or volatilizing the sample. Instruments that we propose would remain on the lander deck to perform analysis include the high-TRL laser desorption mass spectrometry (cf. the Mars Organic Molecule Analyser (MOMA) onboard the upcoming ExoMars Rover (18)). MOMA could provide information on the bulk and isotopic composition of major gases. A microCT should be employed to study the internal structure of the ice.

Planetary Protection: The PLD are not considered special regions under planetary protection definitions; however, a radioisotope thermoelectric generator (RTG) could induce a special region by warming the ice to the melting point, making the landing site Category IV. One method to mitigate this potential without adding significant mass for batteries is to use wind to power the electronics and instruments that are to be operated during the polar night. Solar power is sufficiently abundant in the martian summer to meet the power demands of instruments and a drill.

\section{Conclusions}

In this paper we outline the overarching questions regarding the climate record of Mars and present a landed polar mission concept that is a necessary step for achieving this goal. It is feasible in the upcoming decade with current technology and would lay the groundwork for a mission, in the following decade, that examines $\sim 500 \mathrm{~m}$ of stratigraphy, recording $\sim 1 \mathrm{Myr}$ of Martian climate history. A New Frontiers-class static lander (e.g. ICE-SAG) would assess all important qualities of the materials at the PLD surface and near subsurface $(50 \mathrm{~cm})$ in order to determine the most appropriate techniques for extracting the great climate record in the future.

\section{References:}

https://sharad.psi.edu/ ibsmith/files/WP_Unlocking_the_Climate_Record_References.pdf 